\title{
The microenvironment in breast cancer progression: biology and implications for treatment
}

Andrew E Place ${ }^{1,2,3+}$, Sung Jin Huh ${ }^{4,5,6+}$ and Kornelia Polyak $k^{4,5,6 *}$

\begin{abstract}
Breast cancer comprises a heterogeneous group of malignancies derived from the ductal epithelium. The microenvironment of these cancers is now recognized as a critical participant in tumor progression and therapeutic responses. Recent data demonstrate significant gene expression and epigenetic alterations in cells composing the microenvironment during disease progression, which can be explored as biomarkers and targets for therapy. Indeed, gene expression signatures derived from tumor stroma have been linked to clinical outcomes. There is increasing interest in translating our current understanding of the tumor microenvironment to the development of novel therapies.
\end{abstract}

\section{Introduction}

Breast cancer is the most common malignancy and the leading cause of cancer-related death in women worldwide [1]. Whereas localized disease is largely curable, metastatic or recurrent disease carries a dismal prognosis. The tumor microenvironment is now recognized as an important participant of tumor progression and response to treatment. As a result, there is increasing interest in developing novel therapies targeting the microenvironment, particularly as it relates to invasive and metastatic progression.

The normal breast duct consists of a luminal epithelial cell layer surrounded by myoepithelial cells, which produce and attach to the basement membrane. The breast microenvironment is composed of extracellular matrix (ECM) and numerous stromal cell types, including endothelial and immune cells, fibroblasts, and adipocytes

\footnotetext{
${ }^{\dagger}$ Contributed equally to this manuscript.

*Correspondence: kornelia_polyak@dfci.harvard.edu

${ }^{4}$ Department of Medical Oncology, Dana-Farber Cancer Institute, 450 Brookline

Avenue, D740C, Boston, MA 02215, USA

Full list of author information is available at the end of the article
}

(Figure 1). Early work investigating epithelial-mesenchymal interactions in tissue differentiation demonstrated that embryonic mesenchyme strongly influences the terminal differentiation of both embryonic and adult epithelia [2]. The influence of ECM is also observed in cell culture whereby normal mammary epithelial cells in laminin-rich three-dimensional matrix form acini with a central lumen, become responsive to lactogenic hormones, and are capable of producing milk proteins $[3,4]$. Components of the microenvironment, including macrophages, myoepithelial and endothelial cells, and several ECM molecules, have been shown to play critical roles in mammary duct morphogenesis [5]. Similarly, the tumor microenvironment is increasingly recognized as a major regulator of carcinogenesis [6]. For decades, pathologists have appreciated the wound-like appearance of desmoplastic tumors, including some breast carcinomas. The now-famous assessment by Dvorak that 'tumors are wounds that do not heal' is being redefined at the molecular level as the role of the tumor microenvironment in cancer progression is elucidated [7].

Breast tumors evolve via sequential progression through defined stages, starting with epithelial hyperproliferation and progressing to in situ, invasive, and metastatic carcinomas [8]. Both clinical and experimental data suggest that ductal carcinoma in situ (DCIS) is a precursor of invasive ductal carcinoma (IDC; Figure 2A,B) $[9,10]$. DCIS lesions contain proliferating neoplastic cells confined to the duct (Figures 1B and 2). A critical, but poorly understood, step in breast cancer progression is the transition from in situ to invasive ductal carcinoma, which is defined by the loss of myoepithelial cell layer and basement membrane (Figure 2). The subsequent spread of tumor cells to distant sites results in metastatic disease. Importantly, the tumor microenvironment has been implicated in each of these steps of cancer progression.

\section{Comprehensive molecular profiling of the microenvironment during tumor progression}

In a pioneering study, Allinen and colleagues [11] isolated multiple cell types from normal breast, DCIS and IDC lesions and analyzed their gene expression profiles using 


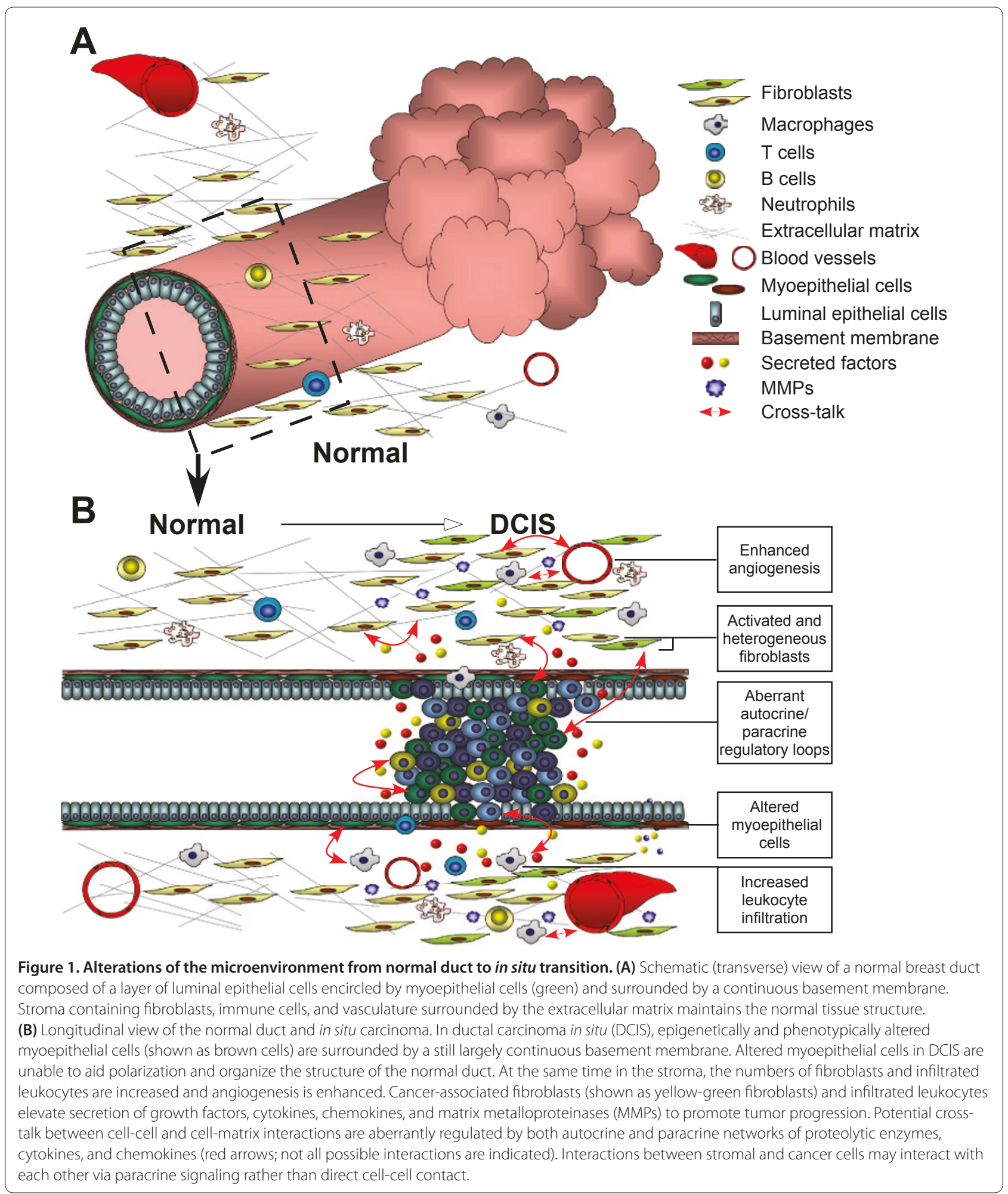

serial analysis of gene expression (SAGE). In addition, genetic changes were detected by cDNA array comprehensive genomic hybridization and single nucleotide polymorphism arrays. The results of this study demonstrated altered gene expression patterns in each cell type analyzed during breast cancer progression. Myoepithelial cells from normal breast tissue and DCIS had the highest number of differentially expressed genes. 


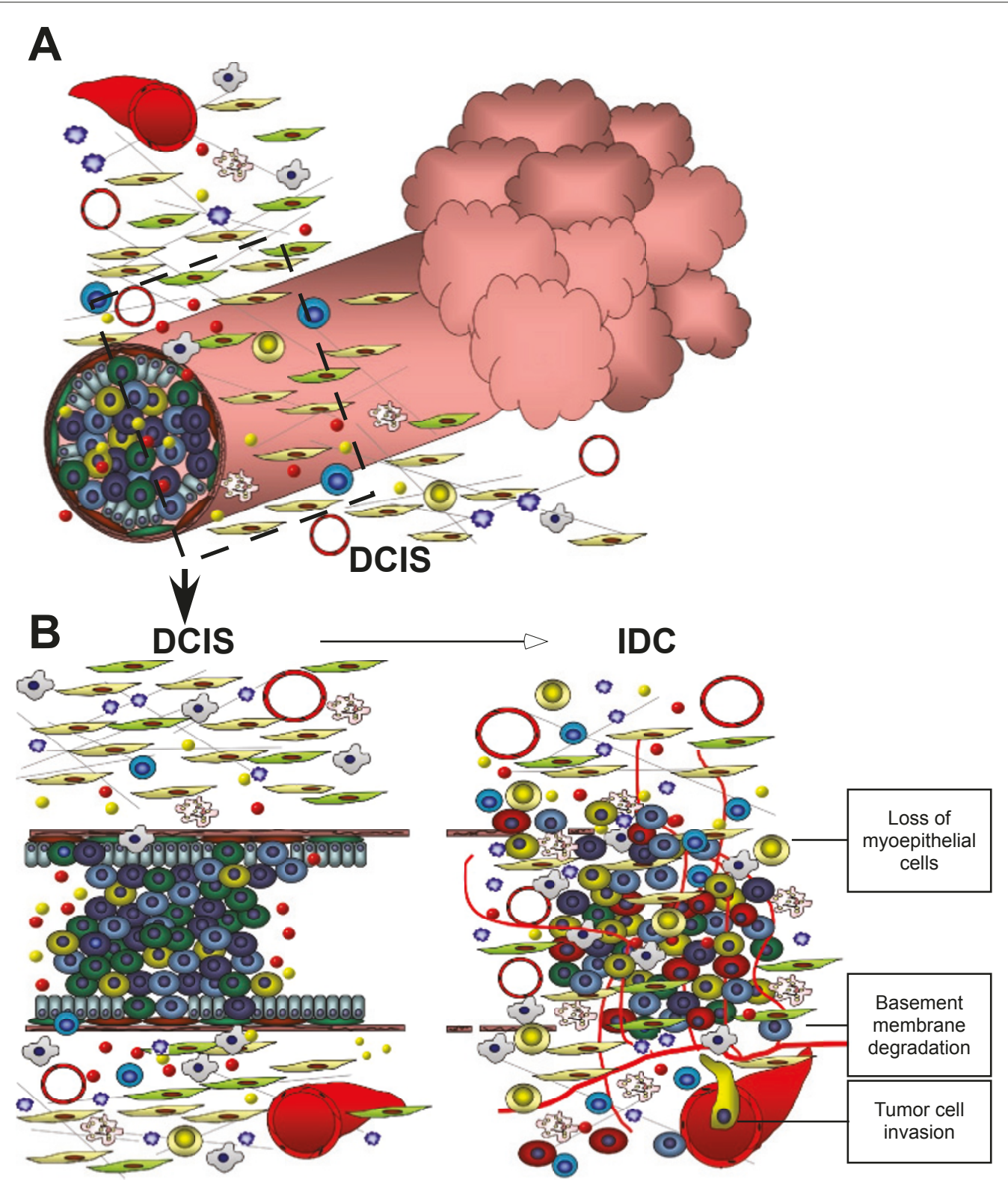

Figure 2. Alterations of the microenvironment in breast cancer progression from in situ to invasive carcinoma. (A) Schematic (transverse) view of the ductal carcinoma in situ (DCIS). Although the ducts are enclosed by the altered myoepithelial cells surrounded by the basement membrane, the multiple cell types of the stroma of DCIS have dramatically changed to create a favorable tumor microenvironment. (B) Longitudinal view of the duct from DCIS to invasive ductal carcinoma transition. Invasive ductal carcinoma (IDC) is defined by degradation of the basement membrane, loss of myoepithelial cells, and invasion of epithelial cells into the stroma and vasculature. Tumor cells invade into the local environment due to the loss of the structural duct and autocrine/paracrine signaling that activated cell migration. The production of extracellular matrix-degrading proteases by the tumor cells and stromal cells is elevated during the in situ to invasive carcinoma transition, leading to destruction of the extracellular matrix such that the tumor cells can invade locally and release more secreted factors. Aberrantly secreted proteolytic enzymes, chemokines, and cytokines continue to attract leukocytes, modulate tumor remodeling, and increase tumor cell invasion to distant organs, eventually leading to metastasis.

A significant fraction of these encode secreted proteins, suggesting the activation of aberrant autocrine and paracrine regulatory loops. Many of the genes involved in normal myoepithelial cell differentiation and function were downregulated in DCIS-associated myoepithelial cells, including those encoding laminin and oxytocin receptor, whereas genes that promote tumorigenesis were increased, including CXCL12 and CXCL14. Importantly, clonal genetic aberrations were only identified within the malignant epithelium. Although some 
controversy remains regarding the existence and relevance of genetically abnormal stromal cells in human breast cancer, the majority of data support the hypothesis that gene expression and functional changes observed in the tumor microenvironment are not due to genetic alterations.

In a related study, Ma and colleagues [12] utilized laser capture microdissection and cDNA microarrays to analyze the gene expression profiles of patient-matched samples of normal- and tumor-associated epithelium (DCIS and IDC) and stroma. Again, the most dramatic gene expression changes both in the stromal and epithelial compartments were observed in the normal to DCIS transition. However, several ECM-degrading proteases, including matrix metalloproteinase (MMP)2 and MMP14, showed elevated expression during in situ to invasive carcinoma transition, which may play a role in the destruction of the basement membrane. One limitation of this study is that the different stromal cells were not individually isolated prior to analysis. Additionally, laser capture microdissection is unable to separate luminal and myoepithelial cells and to fully isolate stroma from epithelium. In fact, the authors noted that the majority of genes with increased expression in IDC compared to DCIS epithelium were likely the result of stromal cell contamination.

Tumor-associated stromal cells maintain their altered phenotype in cell culture during prolonged passage [13], indicating hereditary changes such as epigenetic modifications, as genetic alterations are very rarely detected [14]. $\mathrm{Hu}$ and colleagues [15] tested this hypothesis by analyzing the comprehensive DNA methylation patterns of multiple cell types from normal breast tissue, DCIS, and IDC using methylation-specific digital karyotyping. Significant methylation changes were identified in each cell type during tumor progression. These data imply that epigenetic modifications are at least in part responsible for the altered phenotype of cells composing the microenvironment in breast cancer.

\section{The importance of myoepithelial cells}

The key characteristic of invasive progression is the loss of the myoepithelial cell layer and basement membrane (Figure 2B). Studies utilizing both in vitro co-culture and xenograft models have demonstrated that normal myoepithelial cells inhibit tumor growth. To better characterize the role of myoepithelial and stromal cells in the transition from DCIS to IDC, Hu and colleagues [16] utilized the MCF10DCIS.com xenograft model, which forms DCIS-like lesions that spontaneously progress to IDC with histological and molecular characteristics resembling human lesions. Co-injection of normal myoepithelial cells efficiently suppressed the growth of MCFDCIS xenografts and the transition to IDC whereas fibroblasts had tumor growth and progression-promoting effects. Gene expression profiling and immunohistochemical analysis of luminal epithelial and myoepithelial cells from MCFDCIS xenografts and human breast tissues have identified transforming growth factor $\beta$ (TGF $\beta$ ) and Hedgehog pathways as specifically expressed in myoepithelial cells, implying an important role in these cells. Correlating with this, downregulation of TGFBR2, SMAD4, or GLI1 in MCFDCIS cells resulted in a decrease in myoepithelial cells and enhanced progression to invasion. These studies suggest that the loss of myoepithelial cells promotes DCIS to IDC transition. The mechanism by which this loss occurs in human tumors is unclear. One hypothesis is that the differentiation of myoepithelial progenitors to fully differentiated myoepithelial cells is compromised due to signals emitted by tumor epithelial and stromal cells. The identification and characterization of these paracrine factors may lead to the development of novel therapeutic approaches for the prevention and treatment of invasive breast cancer.

\section{Cancer-associated fibroblasts}

Normal fibroblasts maintain the extracellular environment through the production and remodeling of the ECM. Carcinoma-associated fibroblasts (CAFs) have distinct characteristics and substantial data support a role for CAFs in promoting tumor progression. CAFs are themselves heterogeneous, with a subset of them identified as myofibroblasts expressing alpha smooth muscle actin ( $\alpha$ SMA), others expressing fibroblast activation protein (FAP), desmin, S100A4 protein, and Thy-1 [17].

Orimo and colleagues [13] demonstrated that CAFs promote tumor growth and increase tumor angiogenesis by secretion of stromal derived factor (SDF)-1/CXCL12, which acts in a paracrine fashion to increase tumor cell proliferation via CXCR4. Hepatocyte growth factor (HGF), acting through the c-Met receptor tyrosine kinase, is another CAF-derived factor that has been implicated in promoting tumor progression and metastasis. The paracrine activation of c-Met on tumor cells by HGF increases invasion of experimental DCIS lesions in xenografts [18]. Interestingly, co-culture of normal mammary fibroblasts with breast cancer cells can 'educate' the fibroblasts to secrete HGF and increase their tumor-promoting activities [19].

The origin of carcinoma-associated fibroblasts has been actively investigated and multiple hypotheses have been proposed. One possibility is that they are derived from native interstitial fibroblasts whose phenotype has been modified by persistent aberrant signaling from neighboring tumor epithelial cells. Alternatively, they can be differentiated from bone marrow-derived mesenchymal stem cells that are recruited to the tumor site via endocrine stimulation by tumor-derived factors. This 
hypothesis is supported by the identification of bone marrow-derived cells within tumors from patients who have previously received gender mis-matched allogenic bone marrow transplantation [20], although the recruitment of these cells by itself does not indicate functional relevance. However, a recent study demonstrated that certain xenografts 'instigate' the growth and metastasis of weakly tumorigenic cell lines via the activation and recruitment of bone marrow-derived cells [21]. In particular, granulin-expressing bone marrow-derived cells stimulate both tumor progression and the desmoplastic response of resident fibroblasts. These data as well as data from other labs [22-25] support the hypothesis that tumor-derived signals stimulate the bone marrow to produce and emit cells that promote tumor progression. Thus, targeting bone marrow-derived cells may influence the treatment of both localized and metastatic disease.

\section{Matrix remodeling in tumor progression}

The ECM of breast cancers is markedly abnormal and believed to promote tumor progression. MMPs are a large family of endopeptidases, which are synthesized predominantly by fibroblasts and normally participate in tissue remodeling and wound healing. Besides degrading ECM components, MMPs can also activate chemokines, cytokines, adhesion molecules, and growth factors, which contribute to tumor progression by increasing tumor cell proliferation (such as the release of insulin-like growth factor from ECM by MMP3 and -7) or by promoting angiogenesis (for example, activation of angiogenic factors by MMP1, $-2,-9$, and -14) [26].

Abnormal physical characteristics of breast tumors, such as abnormal collagen cross-linking resulting in ECM stiffening, also contribute to progression. The forces generated by this stiffening lead to enhanced integrin and growth factor signaling that promotes invasion. Lysyl oxidase, an amine oxidase commonly expressed in breast tumors, promotes collagen cross-linking and enhances ECM stiffening and its inhibition increases tumor latency and decreases tumor burden in the MMTV-Neu model of breast cancer [27]. In addition, elevated expression of lysyl oxidase-like 2 is associated with worse prognosis in early stage estrogen receptor (ER)-negative breast cancers [28].

\section{Leukocytes}

The link between inflammation and cancer and the importance of infiltrating leukocytes in tumor development are widely accepted, but the mechanisms mediating immune and tumor cell cross-talk are poorly understood. Immune cells are one of the most dynamic cell populations present within tumors and healing wounds and during the remodeling of breast tissue in pregnancy and involution [29,30]. During physiologic wound healing and breast tissue involution, immune responses are activated, but balanced towards the suppression of overt inflammation, facilitating re-epithelialization and tissue healing $[29,31]$.

High numbers of infiltrating leukocytes are present in DCIS with focal myoepithelial cell layer disruptions [32], suggesting that they might play a role in invasive progression. Indeed, several groups have shown that tumor-associated macrophages (TAMs) facilitate angiogenesis, ECM degradation, and tumor invasion through activation of epidermal growth factor receptor signaling, secretion of proteases and paracrine signaling between tumor cells $[33,34]$. Loss of macrophages in colony stimulating factor (CSF)-1 deficient mice (Csf1 ${ }^{\text {op/op}}$ ) had no effect on tumor initiation but dramatically reduced malignant progression [35]. To determine whether human breast cancer cells have similar response to macrophages, xenografts derived from human MCF-7 cells in immunodeficient mice were treated with either mouse CSF-1 antisense oligonucleotide or CSF-1 small interfering RNAs. These treatments suppressed mammary tumor growth by decreasing macrophage infiltration, the production of MMPs and vascular endothelial growth factor (VEGF)-A, and endothelial cell proliferation [36]. Cumulatively, these functional studies in mouse models of breast cancer emphasize a prominent role for macrophages during breast cancer progression, and provide a plausible explanation for the association between TAMs and poor clinical prognosis.

Besides macrophages, other immune cells have also been implicated in breast cancer development. In a spontaneous mouse model of breast cancer the number of $\mathrm{CD} 4+$ regulatory $\mathrm{T}$ lymphocytes increased and systemic depletion of $\mathrm{T}$ cells using interleukin-2 immunotoxin fusion protein inhibited tumor growth and maintained a strong and persistent anti-tumor immune response [37]. A recent study analyzed tumor-infiltrating CD8+ lymphocytes in breast tumors and found that a higher frequency of these cells in stroma surrounding the tumor was associated with better patient survival [38]. Because these cells are required for cell-mediated immunity, these results may indicate an active anti-tumor immune response against breast tumors, the intensity of which may influence the risk of distant metastatic progression.

\section{The microenvironment of metastases}

Although detailed cellular and molecular characterization of the metastatic tumor microenvironment has not been performed, numerous functional studies support a role for tumor epithelial-stromal cell interactions. During metastatic progression, tumor cells encounter and must survive in a number of different microenvironments, such as blood, lymphatics, lymph nodes, and distant organs. The specific destination where the metastatic cell forms metastases may be mediated by the production of 
chemoattractants, by the local organ and/or the secretion of various products by the primary tumor that can create a favorable environment (Figure 3). Weinberg and colleagues [39] have shown that MDA-MB-231 xenograft tumors growing on one side of the mouse, termed instigator, mobilized bone marrow precursors via secreting osteopontin to home to secondary metastatic sites, where they promoted the growth of a less malignant cell line. Despite the fact that the mechanism by which osteopontin supported metastasis is not clear, this study not only demonstrates the recruitment of bone marrowderived cells to metastatic sites but also highlights the systemic effects of primary tumor growth.

In animal models of breast cancer, $\mathrm{F} 4 / 80^{+} \mathrm{CSF}^{-}$ $1 \mathrm{R}^{+} \mathrm{CD} 11 \mathrm{~b}^{+} \mathrm{Gr} 1{ }^{-} \mathrm{CX} 3 \mathrm{CR} 1^{\text {high }} \mathrm{CCR} 2^{\text {high }}$ and VEGFR $1^{\text {high }}$ host macrophages, distinct from classical macrophages, are recruited to metastatic breast cancer cells extravasating in the lungs [40]. In another study, primary tumor-stimulated macrophages increased the metastatic ability of tail-vein injected tumor cells via secretion of MMP9 and VEGF [41], although lung metastases only developed in mice bearing primary tumors, again indicating systemic effects of tumor growth.

A recent study provided another example of how paracrine signals emitted by stromal cells may play important roles in promoting breast cancer metastasis. Receptor activator of nuclear factor- $\mathrm{KB}$ (RANK) is highly expressed in human breast carcinoma cells, but the source of RANK ligand (RANKL) and its role in breast cancer metastasis was mostly unknown. In a mouse model of ERBB2-driven mammary tumors, Tan and colleagues [42] identified a role for RANKL in the formation of lung metastases. Only $\mathrm{CD} 4^{+}$regulatory $\mathrm{T}$ cells in the stroma of mammary tumors produced RANKL, which stimulated RANK-expressing ERBB2-driven mammary tumors to metastasize. In addition, most RANKL ${ }^{+} \mathrm{T}$ cells were located adjacent to myofibroblasts expressing T-cell-attracting chemokine CCL5. These studies indicate the possibility that recruitment of distinct populations of leukocytes and stromal cells is required in the process of metastasis.

\section{The prognostic relevance of microenvironmental changes}

Interest in targeting the microenvironment comes not only from the identification of 'druggable' targets (enzymes and receptors) but also from clinical data demonstrating that stroma-derived gene expression patterns predict clinical outcome. One of the first such studies, by Wang and colleagues [43], first defined an 'activated fibroblast' gene expression signature that was then analyzed in genome-wide expression data of bulk breast tumor samples from patients with lymph nodenegative disease to determine if it has prognostic relevance. A 76-gene signature was identified that predicted shorter distant metastasis-free survival independent of patient age, tumor size, grade, or ER status. The authors proposed that patients with smaller $(<2 \mathrm{~cm})$ tumors and good prognosis based on their signature may not benefit from adjuvant chemotherapy and could be spared associated morbidities [43]. A related fibroblast signature was developed by Chang and colleagues [44], defined as 'core serum response' genes activated in fibroblasts exposed to serum. Many of the functions of the identified genes were related to wound healing, such as matrix remodeling, myofibroblastic activation, cell proliferation and motility. This gene signature was then validated as a predictor of clinical outcome when applied to expression profiling of whole tumor samples $[44,45]$.

More recently, investigators have focused on breast tumor stroma-derived gene expression changes. Finak and colleagues [46] analyzed gene expression changes within tumor stroma and identified 'outcome-linked' clusters that were independent of tumor grade, size, hormone receptor, and lymph node status. The poor outcome cluster was associated with increased expression of hypoxia- and angiogenesis-related genes, whereas the good outcome cluster was enriched for $\mathrm{T}$ cell immune responses and natural killer cell markers. The authors derived a 26-gene signature that predicted clinical outcome independent of tumor ER or human epidermal growth factor receptor (HER)2 status, implying distinct stromal subtypes distinct of breast tumor subtype. In a related study, Farmer and colleagues [47] analyzed the gene expression profiles of reactive tumor stroma from biopsies obtained prior to treatment of ER-negative tumors and derived a signature that predicted clinical response to neoadjuvant chemotherapy. Similarly, Bergamaschi and colleagues [48] defined several ECM signatures based on gene expression profiling of whole tumor samples that predicted clinical response. In addition to these global profiling studies, individual prognostic markers have also been identified. In DCIS, patients with tumors expressing low levels of CD10 (a myoepithelial cell surface marker) had a higher risk of local relapse [49]. While potentially interesting, small sample size and mixing outcomes from patients treated with mastectomy or lumpectomy limit the validity of the findings. The expression of several CAF-derived proteins is also associated with clinical outcome. For example, high levels of platelet-derived growth factor- $\beta$ receptor or SDF-1/CXCL12 and decreased caveolin-1 are associated with worse clinical outcome [50-52].

\section{Therapeutic targeting of the microenvironment}

New insights into the tumor microenvironment, both focused and global, are identifying novel therapeutic 


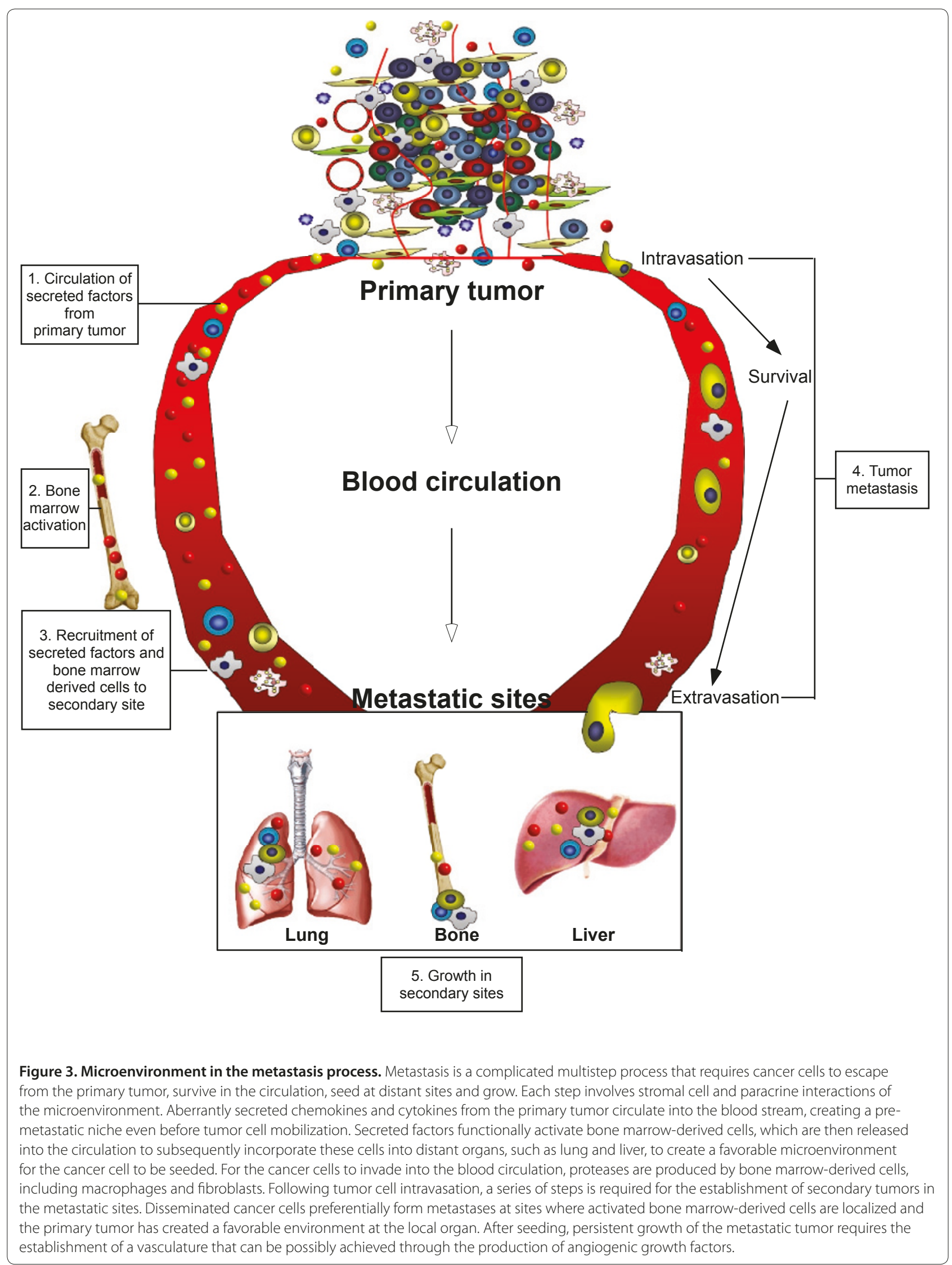


targets. Currently, three types of tumor microenvironment-targeting therapies are in clinical practice: aromatase inhibitors (which target the aromatase enzyme predominantly expressed by stromal components), angiogenesis-modulating agents (including anti-VEGF receptor antagonists), and inhibitors of HER family receptors (such as trastuzumab, which inhibits receptor signaling on epithelial cells triggered by stroma-produced growth factors).

Whereas aromatase inhibitors and trastuzumab have become standard therapy, the clinical effectiveness of angiogenesis inhibitors is less clear [53]. In addition, there is a concern that inhibition of angiogenesis may enhance disease progression based on data in animal models where treatment with anti-angiogenic agents increased invasiveness and metastatic spread [54,55]. Potential selection for hypoxia-tolerant clones or establishment of leaky, metastasis-promoting vessels could explain these results [56]. In addition to these concerns, bevacizumab, a clinically approved VEGF inhibitor, has been associated with significant adverse reactions, including hemorrhage, neutropenia, gastrointestinal perforation, and thromboembolic events. A recently published meta-analysis of 16 randomized controlled clinical trials administering bevacizumab demonstrated that this agent, when used in combination with chemotherapy, was associated with increased risk of fatal treatment-related adverse events compared to the use of chemotherapy alone [57]. Whereas targeting the tumor microenvironment is an exciting possibility, side effects resulting from disruption of homeostatic functions in normal tissues are very likely, as was demonstrated by the poor tolerability of MMP inhibitors. In the past years, numerous targets have been investigated in early clinical trials, including antibodies targeting FAP, c-Met antagonists and multi-targeted receptor tyrosine kinase inhibitors such as sunitinib [58,59]. Some of these have been plagued by poor side effect profiles whereas others have been well tolerated, but ineffective.

In addition to drugs being developed against novel targets, the anti-tumor effects of several older agents seem to be mediated through microenvironmental actions. For example, bisphosphonates (e.g., zoledronic acid), which are used for the treatment of osteoporosis and the management of bone metastasis, are now recognized to have activity outside of the skeleton, including direct anti-tumor effects on the malignant epithelium, and modulating angiogenesis and immune cell infiltration [60].

Osteoclasts are an important component of the normal bone microenvironment as well as bone metastases. Metastatic tumor cells secrete growth factors that activate osteoclasts, which degrade bone and release additional growth factors, triggering a paracrine cascade that promotes tumor growth and bone destruction. Denosumab is a monoclonal antibody that binds RANKL and inhibits osteoclast function. Recently, denosumab was compared to zoledronic acid in a phase III randomized clinical trial in breast cancer patients with bone metastases [61]. The results of this trial demonstrated that denosumab was well tolerated, and superior to zoledronic acid in delaying time to complications of bone metastases (that is, pathological fractures) but did not improve survival.

An important new hypothesis in targeting the tumor microenvironment is the induction of microenvironmental 'reprogramming. Rolny and colleagues [62] recently published an intriguing discovery that overexpression of histidine-rich glycoprotein (HRG) in murine syngeneic tumor models induced 'normalization' of TAMs and blood vessel structure. Importantly, this was associated with decreased breast tumor growth and pulmonary metastasis, and increased sensitivity to chemotherapy. The authors demonstrated that the effects of HRG were dependent on the presence of TAMs and in particular TAM conversion from the 'M2' pro-tumor/ pro-angiogenic phenotype to the 'M1' anti-tumor/proinflammatory phenotype. In addition, HRG expression was associated with vessel normalization, which was also dependent on TAM activity. This study has linked the phenotypic switching of TAMs by HRG with orchestration of vascular normalization. This effect of HRG on TAMs seems to be mediated through the down-regulation of placental growth factor, though the precise mechanism is unclear.

Coussens and colleagues have recently described that the ratio of macrophages to $\mathrm{T}$ cells predicts clinical outcome, with increased macrophage recruitment associated with worse outcome [63]. Interestingly, cytotoxic chemotherapy induces the recruitment of TAMs into invasive carcinomas by increasing the expression of CSF-1, a macrophage-recruiting cytokine. Inhibition of TAM recruitment by several approaches increased the efficacy of chemotherapy by decreasing tumor development and metastasis in a CD8+ T cell-dependent manner. The authors postulate that chemotherapy increases TAM recruitment that subsequently modulates $\mathrm{T}$ cells, favoring the CD4+ T-cell phenotype, which leads to inhibition of anti-tumor immunity. Inhibition of TAMs promotes CD8+ T-cell recruitment and is associated with increased anti-tumor immunity. These data support the development of novel compounds that target TAMs and, in concert with cytotoxic chemotherapy, can encourage anti-tumor immunity [63].

Response to chemotherapy can be assessed by changes in tumor size and imaging characteristics as well as histopathological assessment. Tumor growth can progress, stabilize or regress in response to chemotherapy. In the 
case of a good tumor response characterized by tumor shrinkage, it is possible that the tumor microenvironment actively participates in the tissue remodeling. A simplistic model would be that classic cytotoxic therapies kill tumor cells, which then gives stromal components the opportunity to 'mop up' the necrotic debris. An alternative hypothesis is that the microenvironment, either as a direct effect of chemotherapy or in response to signals derived from the assaulted epithelium, acquires an altered phenotype that independently inhibits tumor growth. Identification of these microenvironmental changes that take place during tumor regression have not been intensively studied. Such studies may identify 'reprogramming' events that can be pharmacologically mimicked with novel, non-cytotoxic agents. Such manipulation of the microenvironment to promote an anti-tumor phenotype in stromal components represents a novel treatment strategy.

Metronomic therapy refers to the frequent or continuous administration of low doses of chemotherapy with the goal of eliciting an anti-tumor response while minimizing side effects. Interestingly, metronomic therapies have been implicated in inhibiting angiogenesis, promoting a beneficial immune response and tumor dormancy [64]. The mechanisms by which metronomic therapies influence these changes are largely unknown. One possible explanation is that these chronic therapies are re-modeling the epigenetic landscape of the tumor microenvironment. Just as the epigenetic changes identified in tumors possibly arise from chronic exposure to pro-tumorigenic signals derived from malignant epithelium, one could postulate a similar affect from chronic exposure to anti-neoplastic agents.

Epigenetic therapies, such as the histone deacetylase inhibitor suberoylanilide hydroxamic acid (also called vorinostat), are currently under clinical investigation for the treatment of breast cancer. While developed to target the malignant epithelium, their effect on the microenvironment may induce alterations that help orchestrate an anti-tumor response. Currently, there are no reports of the gene expression or epigenetic profiles of tumor samples obtained from patients treated with metronomic therapy or histone deacetylase inhibitors. These data will be valuable to our understanding of the microenvironmental changes induced by these therapies.

Besides identifying new therapeutic targets, the microenvironment has also been implicated in chemotherapy resistance. Weaver and colleagues [65], working with three-dimensional cultures, demonstrated that sensitivity to chemotherapy could be influenced by cellular polarity, which is mediated in part by integrin expression and exposure to basement membrane. Hiscox and colleagues [66] demonstrated that resistance to fulvestrant, an antiestrogen, promotes an invasive phenotype secondary to increased epithelial expression of c-MET, which is then activated by fibroblast-produced HGF. Loeffer and colleagues [67] generated an oral vaccine against FAP and studied its effect on the growth of multidrug-resistant breast cancer in murine xenografts. The vaccine decreased tumor collagen I, an ECM component previously implicated in chemotherapy resistance, and tumors from these animals had a significant improvement in chemotherapy uptake as well as decreased tumor growth resulting in increased survival. These data demonstrate that, in addition to promoting progression, the microenvironment can modulate sensitivity or resistance to chemotherapy.

\section{Conclusions and future directions and challenges}

Breast cancer remains a major clinical challenge with considerable mortality as well as treatment-associated morbidity. Novel treatment strategies are urgently needed, especially in the setting of metastatic disease where outcomes are still dismal. The breast cancer microenvironment is a complex mixture of cells, the proteins they secrete, and the ECM in which they reside. Alterations within the microenvironment are now recognized during key steps of tumor progression, making them attractive candidates for therapeutic modulation. The relative genomic stability of stromal cells makes the development of chemoresistance to stromal-target therapy less likely. Furthermore, the epigenetic modifications that contribute to phenotypic alterations, while inheritable, are reversible, and there is mounting interest in 'normalizing' the altered stroma, thereby abrogating its tumor-supporting role.

One major obstacle facing stromal-targeted therapy is avoiding disruption of homeostatic function in normal tissues. Despite these challenges, our improved understanding of key aspects of tumor progression should lead to treatment strategies that can discriminate normal tissue from neoplasm.

How the tumor microenvironment changes during chemotherapy-induced tumor regression is still poorly understood. Insights into these changes may identify important pathways, which can be activated using noncytotoxic therapies. As the mainstay of aggressive forms of breast cancer will continue to rely heavily on cytotoxic therapies for the foreseeable future, agents without these characteristics will be particularly valuable in combination trials.

Translating our burgeoning knowledge of microenvironmental influences on tumor progression into clinical practice is challenging. For example, targeting bone marrow-derived mesenchymal cells, which influence both primary tumor growth and the metastatic niche, prior to clinically evident metastatic disease makes intuitive sense. However, testing these potentially 
important agents in early clinical trials of recurrent or refractory disease may not yield significant improvements in such advanced disease. Thoughtful clinical trial design, including neoadjuvant therapy during which pre- and post-treatment tumor samples can be analyzed, will be vitally important in developing stromal-targeted therapy. Despite these challenges, taken together, the majority of data support the rationale for targeting the tumor microenvironment in the treatment of breast cancer.

\section{Abbreviations}

CAF, carcinoma-associated fibroblast; CSF, colony stimulating factor; DCIS, ductal carcinoma in situ; ECM, extracellular matrix; ER, estrogen receptor; FAP, fibroblast activation protein; HER, human epidermal growth factor receptor; HGF, hepatocyte growth factor; HRG, histidine-rich glycoprotein; IDC, invasive ductal carcinoma; MMP, matrix metalloproteinase; RANK, receptor activator of nuclear factor-KB; RANKL, RANK ligand; SDF, stromal derived factor; TAM, tumor-associated macrophage; VEGF, vascular endothelial growth factor.

\section{Competing interests}

$\mathrm{KP}$ receives research support and is a consultant to Novartis Pharmaceuticals, Inc.; KP is also a member of the scientific advisory boards of Metamark Genetics Inc., and Theracrine Inc., and owns stocks of Aveo Pharmaceuticals Inc.

\section{Acknowledgements}

We thank members of the Polyak laboratory for their critical reading of the manuscript and for helpful discussions.

\section{Author details}

'Department of Pediatric Oncology, Dana-Farber Cancer Institute, 450 Brookline Avenue, D740C, Boston, MA 02215, USA. ²Division of Pediatric Hematology/Oncology, Children's Hospital, 300 Longwood Avenue, Boston, MA 02115, USA. 'Department of Pediatrics, Harvard Medical School, 25 Shattuck Street, Boston, MA 02115, USA. ${ }^{\circ}$ Department of Medical Oncology, Dana-Farber Cancer Institute, 450 Brookline Avenue, D740C, Boston, MA 02215, USA. 'Department of Medicine, Brigham and Women's Hospital, 75 Francis Street, Boston, MA 02115, USA. ${ }^{6}$ Department of Pediatrics, Harvard Medical School, 25 Shattuck Street, Boston, MA 02115, USA.

Published: 1 November 2011

\section{References}

1. Jemal A, Siegel R, Xu J, Ward E: Cancer statistics, 2010. CA Cancer J Clin 2010, 60:277-300

2. Sakakura T, Nishizuka Y, Dawe CJ: Mesenchyme-dependent morphogenesis and epithelium-specific cytodifferentiation in mouse mammary gland. Science 1976, 194:1439-1441.

3. Howlett AR, Bissell MJ: The influence of tissue microenvironment (stroma and extracellular matrix) on the development and function of mammary epithelium. Epithelial Cell Biol 1993, 2:79-89.

4. Petersen OW, Ronnov-Jessen L, Howlett AR, Bissell MJ: Interaction with basement membrane serves to rapidly distinguish growth and differentiation pattern of normal and malignant human breast epithelial cells. Proc Natl Acad Sci U S A 1992, 89:9064-9068. A published erratum appears in Proc Natl Acad Sci U S A 1993, 90:2556.

5. Maller $\mathrm{O}$, Martinson $\mathrm{H}$, Schedin P: Extracellular matrix composition reveals complex and dynamic stromal-epithelial interactions in the mammary gland. J Mammary Gland Biol Neoplasia 2010, 15:301-318.

6. Hanahan D, Weinberg R: Hallmarks of cancer: the next generation. Cell 2011, 144:646-674.

7. Dvorak HF: Tumors: wounds that do not heal. Similarities between tumor stroma generation and wound healing. N Engl J Med 1986, 315:1650-1659.

8. Polyak K: Breast cancer: origins and evolution. J Clin Invest 2007, 117:3155-3163

9. Burstein HJ, Polyak K, Wong JS, Lester SC, Kaelin CM: Ductal carcinoma in situ of the breast. NEngl J Med 2004, 350:1430-1441.

10. Espina $V$, Liotta LA: What is the malignant nature of human ductal carcinoma in situ? Nat Rev Cancer 2011, 11:68-75.
11. Allinen M, Beroukhim R, Cai L, Brennan C, Lahti-Domenici J, Huang H, Porter D, Hu M, Chin L, Richardson A, Schnitt S, Sellers WR, Polyak K: Molecular characterization of the tumor microenvironment in breast cancer. Cancer Cell 2004, 6:17-32.

12. Ma XJ, Dahiya S, Richardson E, Erlander M, Sgroi DC: Gene expression profiling of the tumor microenvironment during breast cancer progression. Breast Cancer Res 2009, 11:R7.

13. Orimo A, Gupta PB, Sgroi DC, Arenzana-Seisdedos F, Delaunay T, Naeem R, Carey VJ, Richardson AL, Weinberg RA: Stromal fibroblasts present in invasive human breast carcinomas promote tumor growth and angiogenesis through elevated SDF-1/CXCL12 secretion. Cell 2005, 121:335-348.

14. Oiu W, Hu M, Sridhar A, Opeskin K, Fox S, Shipitsin M, Trivett M, Thompson ER, Ramakrishna M, Gorringe KL, Polyak K, Haviv I, Campbell IG: No evidence of clonal somatic genetic alterations in cancer-associated fibroblasts from human breast and ovarian carcinomas. Nat Genet 2008, 40:650-655.

15. Hu M, Yao J, Cai L, Bachman KE, van den Brule F, Velculescu V, Polyak K. Distinct epigenetic changes in the stromal cells of breast cancers. Nat Genet 2005, 37:899-905

16. Hu M, Yao J, Carroll DK, Weremowicz S, Chen H, Carrasco D, Richardson A, Violette S, Nikolskaya T, Nikolsky Y, Bauerlein EL, Hahn WC, Gelman RS, Allred C, Bissell MJ, Schnitt S, Polyak K: Regulation of in situ to invasive breast carcinoma transition. Cancer Cell 2008, 13:394-406.

17. Xing F, Saidou J, Watabe K: Cancer associated fibroblasts (CAFs) in tumor microenvironment. Front Biosci 2010, 15:166-179.

18. Jedeszko C, Victor BC, Podgorski I, Sloane BF: Fibroblast hepatocyte growth factor promotes invasion of human mammary ductal carcinoma in situ. Cancer Res 2009, 69:9148-9155.

19. Tyan SW, Kuo WH, Huang CK, Pan CC, Shew JY, Chang KJ, Lee EY, Lee WH: Breast cancer cells induce cancer-associated fibroblasts to secrete hepatocyte growth factor to enhance breast tumorigenesis. PLoS One 2011, 6:e15313.

20. Worthley DL, Ruszkiewicz A, Davies R, Moore S, Nivison-Smith I, Bik To L, Browett P, Western R, Durrant S, So J, Young GP, Mullighan CG, Bardy PG, Michael MZ: Human gastrointestinal neoplasia-associated myofibroblasts can develop from bone marrow-derived cells following allogeneic stem cell transplantation. Stem Cells 2009, 27:1463-1468.

21. Elkabets M, Gifford AM, Scheel C, Nilsson B, Reinhardt F, Bray MA, Carpenter $A E$, Jirström K, Magnusson K, Ebert BL, Pontén F, Weinberg RA, McAllister SS: Human tumors instigate granulin-expressing hematopoietic cells that promote malignancy by activating stromal fibroblasts in mice. J Clin Invest 2011, 121:784-799.

22. Shin SY, Nam JS, Lim Y, Lee YH: TNFalpha-exposed bone marrow-derived mesenchymal stem cells promote locomotion of MDA-MB-231 breast cancer cells through transcriptional activation of CXCR3 ligand chemokines. J Biol Chem 2010, 285:30731-30740.

23. Goldstein $\mathrm{RH}$, Reagan MR, Anderson $\mathrm{K}$, Kaplan DL, Rosenblatt M: Human bone marrow-derived MSCs can home to orthotopic breast cancer tumors and promote bone metastasis. Cancer Res 2010, 70:10044-10050.

24. Karnoub AE, Dash AB, Vo AP, Sullivan A, Brooks MW, Bell GW, Richardson AL, Polyak K, Tubo R, Weinberg RA: Mesenchymal stem cells within tumour stroma promote breast cancer metastasis. Nature 2007, 449:557-563.

25. Direkze NC, Jeffery R, Hodivala-Dilke K, Hunt T, Playford RJ, Elia G, Poulsom R, Wright NA, Alison MR: Bone marrow-derived stromal cells express lineagerelated messenger RNA species. Cancer Res 2006, 66:1265-1269.

26. Chabottaux V, Noel A: Breast cancer progression: insights into multifaceted matrix metalloproteinases. Clin Exp Metastasis 2007, 24:647-656.

27. Levental KR, Yu H, Kass L, Lakins JN, Egeblad M, Erler JT, Fong SF, Csiszar K, Giaccia A, Weninger W, Yamauchi M, Gasser DL, Weaver VM: Matrix crosslinking forces tumor progression by enhancing integrin signaling. Cell 2009, 139:891-906.

28. Barker HE, Chang J, Cox TR, Lang G, Bird D, Nicolau M, Evans HR, Gartland A, Erler JT: LOXL2-mediated matrix remodeling in metastasis and mammary gland involution. Cancer Res 2011, 71:1561-1572.

29. Coussens LM, Werb Z: Inflammation and cancer. Nature 2002, 420:860-867.

30. McDaniel SM, Rumer KK, Biroc SL, Metz RP, Singh M, Porter W, Schedin P: Remodeling of the mammary microenvironment after lactation promotes breast tumor cell metastasis. Am J Pathol 2006, 168:608-620.

31. de Visser KE, Eichten A, Coussens LM: Paradoxical roles of the immune system during cancer development. Nat Rev Cancer 2006, 6:24-37.

32. Man YG, Sang QX: The significance of focal myoepithelial cell layer 
disruptions in human breast tumor invasion: a paradigm shift from the "protease-centered" hypothesis. Exp Cell Res 2004, 301:103-118.

33. DeNardo DG, Barreto JB, Andreu P, Vasquez L, Tawfik D, Kolhatkar N, Coussens LM: CD4(+) T cells regulate pulmonary metastasis of mammary carcinomas by enhancing protumor properties of macrophages. Cancer Cell 2009, 16:91-102.

34. Schedin P, O'Brien J, Rudolph M, Stein T, Borges V: Microenvironment of the involuting mammary gland mediates mammary cancer progression. J Mammary Gland Biol Neoplasia 2007, 12:71-82.

35. Lin EY, Nguyen AV, Russell RG, Pollard JW: Colony-stimulating factor 1 promotes progression of mammary tumors to malignancy. J Exp Med 2001, 193:727-740.

36. Aharinejad S, Paulus P, Sioud M, Hofmann M, Zins K, Schafer R, Stanley ER, Abraham D: Colony-stimulating factor-1 blockade by antisense oligonucleotides and small interfering RNAs suppresses growth of human mammary tumor xenografts in mice. Cancer Res 2004, 64:5378-5384.

37. Knutson KL, Dang Y, Lu H, Lukas J, Almand B, Gad E, Azeke E, Disis ML: IL-2 immunotoxin therapy modulates tumor-associated regulatory $T$ cells and leads to lasting immune-mediated rejection of breast cancers in neutransgenic mice. J Immuno/ 2006, 177:84-91.

38. Mahmoud SM, Paish EC, Powe DG, Macmillan RD, Grainge MJ, Lee AH, Ellis IO, Green AR: Tumor-infiltrating CD8+ lymphocytes predict clinical outcome in breast cancer. J Clin Oncol 2011, 29:1949-1955.

39. McAllister SS, Gifford AM, Greiner AL, Kelleher SP, Saelzler MP, Ince TA, Reinhardt F, Harris LN, Hylander BL, Repasky EA, Weinberg RA: Systemic endocrine instigation of indolent tumor growth requires osteopontin. Cell 2008, 133:994-1005

40. Qian B, Deng Y, Im JH, Muschel RJ, Zou Y, Li J, Lang RA, Pollard JW: A distinct macrophage population mediates metastatic breast cancer cell extravasation, establishment and growth. PLoS One 2009, 4:e6562.

41. Hiratsuka S, Nakamura K, Iwai S, Murakami M, Itoh T, Kijima H, Shipley JM, Senior RM, Shibuya M: MMP9 induction by vascular endothelial growth factor receptor-1 is involved in lung-specific metastasis. Cancer Cell 2002, 2:289-300.

42. Tan W, Zhang W, Strasner A, Grivennikov S, Cheng JQ, Hoffman RM, Karin M: Tumour-infiltrating regulatory $T$ cells stimulate mammary cancer metastasis through RANKL-RANK signalling. Nature 2011, 470:548-553.

43. Wang Y, Klijn JG, Zhang Y, Sieuwerts AM, Look MP, Yang F, Talantov D, Timmermans M, Meijer-van Gelder ME, Yu J, Jatkoe T, Berns EM, Atkins D, Foekens JA: Gene-expression profiles to predict distant metastasis of lymph-node-negative primary breast cancer. Lancet 2005, 365:671-679.

44. Chang HY, Sneddon JB, Alizadeh AA, Sood R, West RB, Montgomery K, Chi JT, van de Rijn M, Botstein D, Brown PO: Gene expression signature of fibroblast serum response predicts human cancer progression: similarities between tumors and wounds. PLoS Biol 2004, 2:E7.

45. Chang HY, Nuyten DS, Sneddon JB, Hastie T, Tibshirani R, Sørlie T, Dai H, He YD, van't Veer $L$, Bartelink $H$, van de Rijn M, Brown PO, van de Vijver MJ: Robustness, scalability, and integration of a wound-response gene expression signature in predicting breast cancer survival. Proc Natl Acad Sci USA 2005, 102:3738-3743

46. Finak G, Bertos N, Pepin F, Sadekova S, Souleimanova M, Zhao H, Chen H, Omeroglu G, Meterissian S, Omeroglu A, Hallett M, Park M: Stromal gene expression predicts clinical outcome in breast cancer. Nat Med 2008, 14:518-527.

47. Farmer $P$, Bonnefoi $H$, Anderle $P$, Cameron D, Wirapati $P$, Becette $V$, André $S$, Piccart M, Campone M, Brain E, Macgrogan G, Petit T, Jassem J, Bibeau F, Blot E, Bogaerts J, Aguet M, Bergh J, Iggo R, Delorenzi M: A stroma-related gene signature predicts resistance to neoadjuvant chemotherapy in breast cancer. Nat Med 2009, 15:68-74.

48. Bergamaschi A, Tagliabue E, Sørlie T, Naume B, Triulzi T, Orlandi R, Russnes HG, Nesland JM, Tammi R, Auvinen P, Kosma VM, Ménard S, Børresen-Dale AL: Extracellular matrix signature identifies breast cancer subgroups with different clinical outcome. J Pathol 2008, 214:357-367.

49. Toussaint J, Durbecq V, Altintas S, Doriath V, Rouas G, Paesmans M, Bedard P, Haibe-Kains B, Tjalma WA, Larsimont D, Piccart M, Sotiriou C: Low CD10 mRNA expression identifies high-risk ductal carcinoma in situ (DCIS). PLoS One 2010, 5:e12100

50. Paulsson J, Sjoblom T, Micke P, Ponten F, Landberg G, Heldin CH, Bergh J, Brennan DJ, Jirstrom K, Ostman A: Prognostic significance of stromal platelet-derived growth factor beta-receptor expression in human breast cancer. Am J Pathol 2009, 175:334-341.

51. Witkiewicz AK, Dasgupta A, Sotgia F, Mercier I, Pestell RG, Sabel M, Kleer CG, Brody JR, Lisanti MP: An absence of stromal caveolin-1 expression predicts early tumor recurrence and poor clinical outcome in human breast cancers. Am J Pathol 2009, 174:2023-2034.

52. Kang H, Watkins G, Parr C, Douglas-Jones A, Mansel RE, Jiang WG: Stromal cell derived factor-1: its influence on invasiveness and migration of breast cancer cells in vitro, and its association with prognosis and survival in human breast cancer. Breast Cancer Res 2005, 7:R402-410.

53. Alvarez RH: Present and future evolution of advanced breast cancer therapy. Breast Cancer Res 2010, 12 Suppl 2:S1.

54. Ebos JM, Lee CR, Cruz-MunozW, Bjarnason GA, Christensen JG, Kerbel RS: Accelerated metastasis after short-term treatment with a potent inhibitor of tumor angiogenesis. Cancer Cell 2009, 15:232-239.

55. Paez-Ribes M, Allen E, Hudock J, Takeda T, Okuyama H, Vinals F, Inoue M, Bergers G, Hanahan D, Casanovas O: Antiangiogenic therapy elicits malignant progression of tumors to increased local invasion and distant metastasis. Cancer Cell 2009, 15:220-231.

56. Loges S, Mazzone M, Hohensinner P, Carmeliet P: Silencing or fueling metastasis with VEGF inhibitors: antiangiogenesis revisited. Cancer Cell 2009, 15:167-170.

57. Ranpura V, Hapani S, Wu S: Treatment-related mortality with bevacizumab in cancer patients: a meta-analysis. JAMA 2011, 305:487-494.

58. Allen M, Louise Jones J: Jekyll and Hyde: the role of the microenvironment on the progression of cancer. J Pathol 2011, 223:162-176.

59. Joyce JA: Therapeutic targeting of the tumor microenvironment. Cancer Cell 2005, 7:513-520.

60. Holen I, Coleman RE: Anti-tumour activity of bisphosphonates in preclinical models of breast cancer. Breast Cancer Res 2010, 12:214.

61. Stopeck AT, Lipton A, Body JJ, Steger GG, Tonkin K, de Boer RH, Lichinitser M, Fujiwara Y, Yardley DA, Viniegra M, Fan M, Jiang Q, Dansey R, Jun S, Braun A: Denosumab compared with zoledronic acid for the treatment of bone metastases in patients with advanced breast cancer: a randomized, double-blind study. J Clin Oncol 2010, 28:5132-5139.

62. Rolny C, Mazzone M, Tugues S, Laoui D, Johansson I, Coulon C, Squadrito ML, Segura I, Li X, Knevels E, Costa S, Vinckier S, Dresselaer T, Åkerud P, De Mol M, Salomäki H, Phillipson M, Wyns S, Larsson E, Buysschaert I, Botling J, Himmelreich U, Van Ginderachter JA, De Palma M, Dewerchin M, ClaessonWelsh L, Carmeliet P: HRG inhibits tumor growth and metastasis by inducing macrophage polarization and vessel normalization through downregulation of PIGF. Cancer Cell 2011, 19:31-44.

63. DeNardo D, Brennan DJ, Rexhepaj E, Ruffell B, Shiao SL, Madder SF, W.M. G, Wadhwani N, Keil SD, Junaid SA, Rugo HS, Hwang ES, Jirström K, West BL, Coussens LM: Leukocyte complexity predicts breast cancer survival and functionally regulates response to chemotherapy. Cancer Disc 2011. doi:10.1158/2159-8274.CD-10-0028.

64. Pasquier E, Kavallaris M, Andre N: Metronomic chemotherapy: new rationale for new directions. Nat Rev Clin Oncol 2010, 7:455-465.

65. Weaver VM, Lelievre S, Lakins JN, Chrenek MA, Jones JC, Giancotti F, Werb Z, Bissell MJ: beta4 integrin-dependent formation of polarized threedimensional architecture confers resistance to apoptosis in normal and malignant mammary epithelium. Cancer Cell 2002, 2:205-216.

66. Hiscox S, Jordan NJ, Jiang W, Harper M, McClelland R, Smith C, Nicholson RI: Chronic exposure to fulvestrant promotes overexpression of the c-Met receptor in breast cancer cells: implications for tumour-stroma interactions. Endocr Relat Cancer 2006, 13:1085-1099.

67. Loeffler M, Kruger JA, Niethammer AG, Reisfeld RA: Targeting tumorassociated fibroblasts improves cancer chemotherapy by increasing intratumoral drug uptake. J Clin Invest 2006, 116:1955-1962.

\section{doi:10.1186/bcr2912}

Cite this article as: Place $A E$, et al:: The microenvironment in breast cancer progression: biology and implications for treatment. Breast Cancer Research 2011, 13:227. 